\title{
Problem-solving in caregiver-counselling (PLiP Study): study protocol of a cluster randomized pragmatic trial
}

\author{
Klaus Pfeiffer ${ }^{* *}$ D, Martin Hautzinger ${ }^{2}$, Margarete Patak², Julia Grünwald ${ }^{2}$, Clemens Becker $^{1}$ and Diana Albrecht ${ }^{1}$
}

\begin{abstract}
Background: Despite the positive evaluation of various caregiver interventions over the past 3 decades, only very few intervention protocols have been translated to delivery in service contexts. The purpose of this study is to train care counsellors of statutory long term care insurances in problem-solving and to evaluate this approach as an additional component in the statutory care counselling in Germany.

Methods: A pragmatic cluster randomized controlled trial in which 38 sites with 58 care counsellors are randomly assigned to provide either routine counselling plus additional problem-solving for caregivers or routine counselling alone. The counsellor training comprises an initial 2-day training, a follow-up day after 4 months, and biweekly supervision contacts with a psychotherapist for 6 months over the phone. The agreed minimum counselling intensity is one initial face-to-face contact including a caregiver assessment and at least one telephone follow-up contact. Caregivers who are positively screened for significant strain in their role are followed up at 3 and 6 months after baseline assessment. Main outcome are caregivers' depressive symptoms.

Discussion: While it is unclear if the expected very low amount of additional counselling time is sufficient to yield any additional effects on caregiver depression, it is also unclear if the additional problem-solving component yields to synergies with routine counselling that is based on information and case management. There are different potential individual and organisational barriers to a consistent intervention delivery like gratification for participation, time for extra work or internal motivation to participate.
\end{abstract}

Trial registration: (ISRCTN23635523)

Keywords: Caregiver, Counselling, Depression, Translational research, Problem-solving

\section{Background}

Approximately 1.86 million $(82.7 \%$ of them are $\geq 60$ years old) out of 2.6 million care recipients in Germany are living at home. Within this group, the majority (1.25 million) receives support exclusively from informal caregivers without using any professional services [1]. Caregiver burden or distress is common [2] and associated with female gender, low education, residence with the care recipient, higher number of hours spent caregiving, depression, social isolation, financial stress, and the lack

\footnotetext{
* Correspondence: klaus.pfeiffer@rbk.de

'Department of Clinical Gerontology and Rehabilitation,

Robert-Bosch-Hospital, Auerbachstr. 110, 70376 Stuttgart, Germany

Full list of author information is available at the end of the article
}

of choice being a caregiver [3]. Interventions to support informal caregivers have been evaluated in diverse disease specific contexts like dementia or stroke [4, 5]. Major components of interventions are psychoeducation, supportive interventions, psychotherapy, respite/ day-care, or training of the care recipient [6]. The interventions are delivered in various ways (e.g. face-to-face or by telephone) and during different stages of the caregiving trajectory. Major endpoints e.g. in dementia caregiver research are depressive symptoms and emotional distress, burden, self-efficacy and coping, and quality of life [7]. Most of the evaluated heterogeneous interventions for dementia caregivers were effective to some extent [4], with a robust corpus of intervention studies 
that demonstrates small but clinically and statistically significant benefits for families [8]. Despite the evaluation of more than 200 dementia caregiver interventions over the past three decades, only very few programs have been translated to delivery in service contexts [9]. These rare efforts demonstrate the multiple challenges between required modifications according to staff and organization needs and the preservation of the integrity of the particular intervention [10].

Over the last years, the German legislature has initiated several new laws within the long-term care act to support care recipients and their caregivers. One regulation (social act / \$7a SGB XI) that became effective in 2009 specifies the right for care recipients to get counselling and support by care counsellors (German: "Pflegeberater"). The counsellors usually have qualifications in the field of social work, nursing, or social insurance plus a defined specific training in nursing knowledge, case management, and legislation. The scope of counselling in this context should range from information transfer to case management. Care plans should be made in accordance with family caregivers who (and not the care recipient as insured person) is in most consultations the counsellor's contact person. Recent laws and counselling practice do not consider caregivers as advice seekers with own needs and interests in a systematic way. While some long term care (LTC) insurances have explicitly addressed caregiver issues in their draft papers or even assessments, the current counselling practice is (to our knowledge) not directly linked to the corpus of evidence-based interventions in this field so far. The first evaluation report from 2012 summarizes that the professional qualification of care counsellors has made good progress, but the ways of implementation are very heterogeneous with regard to insurances, local districts, and states. Burden, mental or physical health outcomes of caregivers, as well as aspects of quality of care were not considered in this national evaluation [11].

From 2007 to 2010 we conducted a randomized controlled trial (ISRCTN86289718) to evaluate the impact of a mostly telephone-based problem-solving (PS) intervention to distressed family caregivers of German geriatric stroke survivors. The intervention's effectiveness on depressive symptoms and physical complaints of the caregivers after the main intervention period (month 3) and the maintenance period (month 12) compared to a control group has significantly been proven [12]. Recent reviews on stroke [13] and dementia [14] caregiver research can underline positive effects of problem-solving interventions for this target group. The prevailing conceptualization of problem solving articulated by D'Zurilla and Nezu [15] describes PS as a general coping strategy with reciprocal relations to stressful life events and emotional stress responses.
The aim of the current study (2013 to 2016) is to train counsellors from nursing care insurances to apply problem-solving as a caregiver specific component and part of an extended care counselling. In a pragmatic trial we want to evaluate the effect of this extension compared to routine counselling on distressed caregivers. In line with the mixed samples of caregivers who receive counselling from LTC insurances, we want to include caregivers of care recipients with all kind of diagnoses (e.g. dementia, stroke, and heart failure). This is in contrast to most of the previous caregiver research that is segmented along different care recipient diagnoses and funding schemes. For the evaluation a cluster randomized design was chosen to prevent contamination of routine counselling of untrained counsellors by improved counselling skills of trained colleagues within the same office. Furthermore, we wanted to prevent that each participating counsellor would have had to switch between two different counselling approaches depending on the group allocation of the caregiver.

\section{Methods \\ Design of the study}

"Problem-solving in caregiver counselling" (German acronym: PLiP - ProblemLösen in der Pflegeberatung) is a translational study with a prospective cluster randomized design. Clusters are district offices of the participating nursing insurances with usually one care counsellor per office. But depending on the insurance and district size, some local offices with more than one counsellor are also included. The clusters were randomly assigned to the problem-solving training group and a control group (Fig. 1). The counsellors of the control group receive the training after finishing the evaluation. The allocation ratio was 1:1. We used a stratified block randomisation grouping district offices into strata defined by number of counsellors per office, and performing block randomisation within each stratum. The computer-generated, controlled random allocation of each local office is provided by an independent randomization centre at the University of Ulm and performed stepwise for each of the three participating insurances after entering the study.

The aim of this trial is to evaluate the impact of an advanced training in problem-solving for care counsellors on family caregivers who experience burden and depressive symptoms compared to usual counselling.

\section{Participants}

\section{Caregiver counsellors}

Recruitment criteria on site of the caregiver counsellors are: 1) providing caregiver counselling in line with the German Social Security Code (\$7a SGB XI), 2) qualification for counselling according to the recommendations of the National Association of Statutory Health 


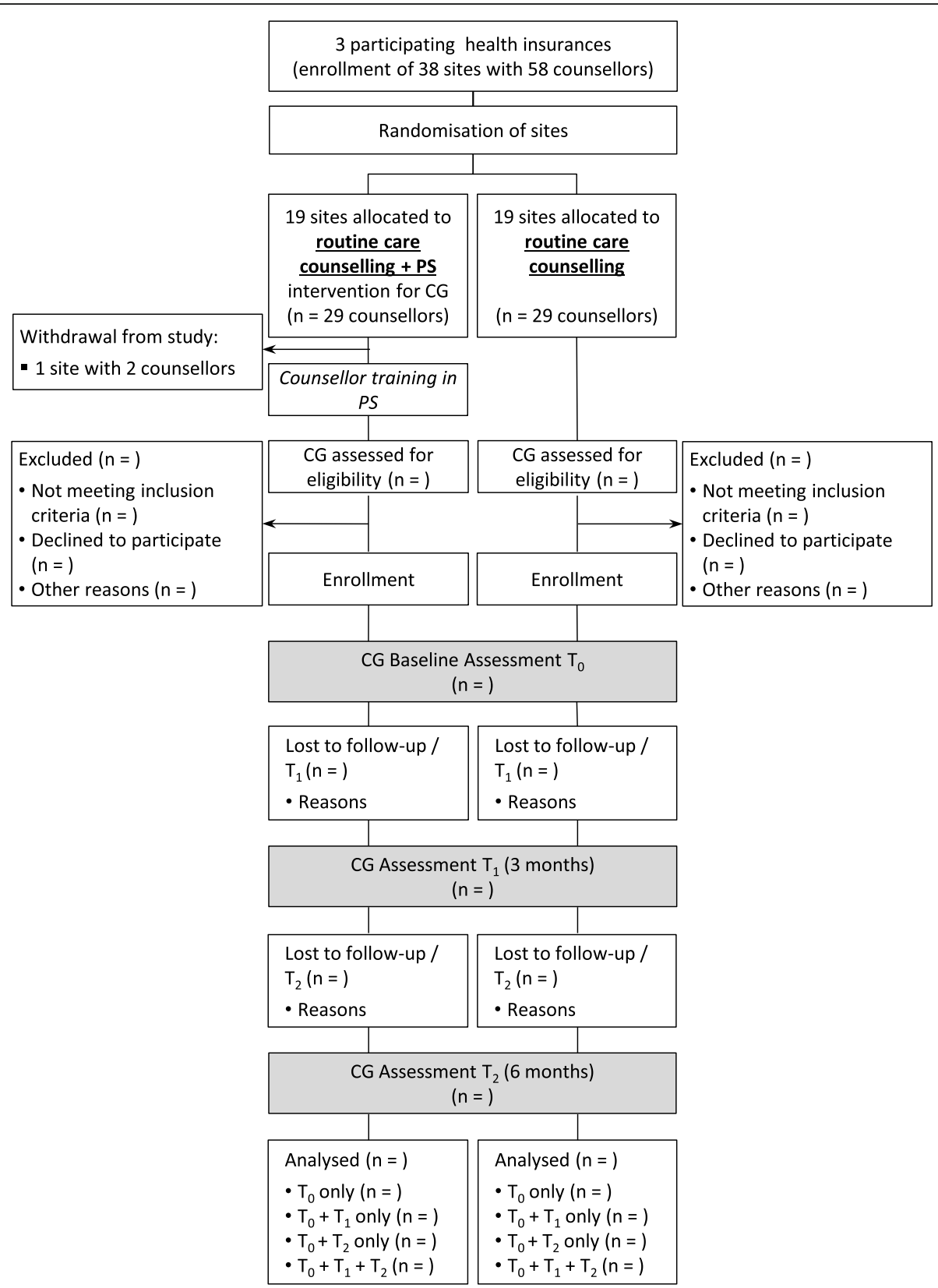

Fig. 1 Recruitment and participation flow chart of care counsellors and caregivers

Insurance Funds, 3) agreement to participate in the advanced training and evaluation of this study. Counsellors are excluded when their participation in the training course is incomplete (non-participation in the 2-day main training course, or/and less than 6 of the 13 biweekly supervision telephone contacts).

\section{Informal caregivers}

Inclusion criteria for informal caregivers are: 1) report distress associated with caregiving (endorsing at least two of three items asking if they feel their mental or physical health has declined due to caregiving tasks, feel lonely during the last week, or experience moderate to high levels of general stress), 2) comprehensive counselling or case management is necessary (at least one personal counselling session and at least one telephonebased or personal follow-up contact are planned), 3) having the primary responsibility for someone who is dependent on care according to the criteria of the German statutory nursing insurance, 4) is the main contact person for the care counsellor, 5) $\geq 18$ years, and 6) consented to participate in the study. Exclusion criteria are: 1) professional paid responsibility for the care recipient, and 2) inability to speak and read German. 


\section{Recruitment of care counsellors and caregivers}

Care counsellors are recruited from three German LTC insurances. The realization of the project is organized together with the person of each insurance who is responsible for care counselling. Depending on the specific LTC insurance, the participation of the counsellors within the project is either by choice or appointed by a principal. All counsellors do not receive extra money or time budgets for participating in the study.

Six informal caregivers should be recruited by each counsellor. For this purpose, caregivers who agreed should be screened consecutively by the counsellors for subjective caregiver burden. In case of a positive screening, the counsellor informs the caregiver about the basic facts of the study and asks for the caregiver's consent to forward his contact details to the study evaluation team. The evaluation team informs about further details of the study to enable the caregiver to make an informed decision whether to participate in the study or not. The baseline assessment must be finished not later than 3 weeks after screening. Family caregivers receive either regular or advanced counselling by the participating counsellors. However, they are blinded and do not know whether their counsellor pertains to the intervention or control group.

\section{Intervention}

The counsellors randomized to the intervention condition receive a specific training on PS that is based on the six principles of the PS model developed by D'Zurilla and colleagues [15-17]: (a) optimism and orientation, (b) problem definition and facts, (c) goal setting, (d) generation of alternatives, (e) decision making, and (f) implementation and verification. The training in PS in our study addresses three facets: (1) Facilitating the caregiver PS as the major component of the training. For problem identification, we trained the counsellors in using a card-sorting task with 37 cards covering possible (care related) problems. The used cards are based on previous research $[12,18,19]$ and modified together with participating care counsellors for this study. As additional materials, three worksheets are provided to support the PS process (situation analyses based on the $\mathrm{ABC}$ model (A $=$ adversity, $\mathrm{B}=$ beliefs, $\mathrm{C}=$ consequences [20]); goal definition, solution generation and evaluation; solution implementation and verification). (2) Using short presentations, small group discussions, and role-plays we apply the described PS steps to improve or overcome difficult interactions between counsellors and caregivers. (3) Other aspects of work-related difficulties or mental hygiene are mainly addressed during the individual telephone contacts. The training is delivered in an initial 2day workshop, a follow-up day after 4 months, and individual bi-weekly telephone supervision contacts over
6 months after the initial workshop to facilitate the implementation of the PS principles and the card-sorting task in daily counselling practice.

While the on-site counsellor trainings in small groups (maximum 12 counsellors) are highly structured, the telephone contacts focus on implementing PS for caregivers in daily routine but also include a tailored educational component based on the counsellor's individual needs and wishes. Possible topics are coping with stressors (e.g. role stress, coping with labor conditions, organization of work, coping with death and dying) and specific strategies and techniques (e.g. resource activation, work-life balance, self-distancing, self-awareness and mindfulness-based techniques). Each supervisor has a list with potential relevant stressors and techniques to facilitate this training component that was implemented not least because to offer the counsellors something they can benefit directly.

The two trainers and supervisors are cognitive behavioral therapists without specific previous experience in caregiver research or care counsellor training. Both are paid on fee basis. Together with at least one of the supervisors, the principal investigator of the study (KP) is involved in the group trainings and has regular meetings with both supervisors.

After the 6-month training the counsellor should use the PS approach including the card-sorting task as an additional component of the usual care counselling $(\mathbb{\$} 7 \mathrm{a}$ SGB XI) with distressed caregivers who are included in the study. The PS steps should be delivered in one initial face-to-face contact (including the card-sorting task) and at least one telephone follow-up contact. During the evaluation period over 6 months the counsellors have four additional contacts with their supervisor. Further contacts with the supervisors are possible if needed.

In addition to the specific counselling, participating caregivers in the intervention group receive five standard information letter (LTC insurance, relief of caregiver strain, depression, problem-solving, relaxation) and if desired up to five more specific letters (nutrition, coping with problem behavior, fall prevention, pain, oral hygiene) by post within 6 month.

\section{Control group}

The caregivers who were recruited by the counsellors of the control group receive advice in the context of the statutory care counselling ( $\$ 7$ a SGB XI).

All care recipients and caregivers of the intervention and control group are getting additional mandatory counselling visits (in line with the German Social Security Code $\$$ 37.3 SGB XI) when receiving only lump-sum transfers and no non-cash benefits like professional support paid by the LTC insurance. 


\section{Evaluation and outcomes}

The project is evaluated on two different levels: 1) impact of the extended care counselling on the informal caregivers, and the training 2) on the care counsellors.

\section{Primary outcome measure}

The primary outcome measure is depressive symptoms of the informal caregiver assessed by the 20-item Centre for Epidemiological Studies Depression scale (CES-D) $[21,22]$ at $T_{0}$ (after enrollment), $T_{1}$ (3 months after $\left.T_{0}\right)$, and $\mathrm{T}_{2}\left(6\right.$ months after $\left.\mathrm{T}_{0}\right)$. Total scores range from 0 to 60 with a score of $\geq 16$ as an indicator of significant symptoms [23]. Table 1 provides an overview of the primary and secondary outcomes that will be measured at the three time points.

\section{Secondary outcome measures Informal caregivers}

Caregiver burden is assessed by a short version [24] of the Sense of Competence Questionnaire (SCQ) [25]. It contains 16 items that are rated on a five-point scale with higher scores indicating a greater sense of competence. Total scores range from 16 to 80 . Subjective physical complaints are assessed by the Giessen Subjective Complaints List (GBB-24) - subscale 'Pains in Limbs' [26] that addresses six symptoms like headache, backache, sense of heaviness and fatigue in legs. The intensity of each of the six complaints is rated on a five-point scale, ranging from 0 (not existing) to 4 (strong). The subscale 'Negative Problem Orientation' of the Social Problem Solving Inventory - Revised (SPSI-R) is used to measure negative problem orientation $[27,28]$. This subscale consists of five items that are rated on a five-point scale ranging from 0 (not very true of me) to 4 (extremely true of me). The total score ranges from 0 to 20 .
Besides, leisure time satisfaction is assessed by the Leisure Time Satisfaction Questionnaire (LTS) [29]. Items are rated on a Likert-type scale ranging from 0 (not at all) to 2 (a lot). The total score ranges from 0 to 12 , and higher scores reflect greater satisfaction. In the validation study [29] the median of the LTS was 5 in a sample of 1,229 dementia caregivers (mean age $=62.3$ years).

Further variables are the caregiver's care-related quality of life (CarerQol) [30], informal care activities, and semi-structured qualitative interviews with a random sample of caregivers.

Additionally, some care recipient characteristics are assessed. Functional disability is measured with the Barthel Index of ADL (BI) [31], higher cognitive functioning (comprehension, verbal expression, social interaction, problem solving, memory/learning/orientation, and vision/neglect) with the Extended Barthel Index (EBI) [32]. Besides, retrospective data on health service use and the amount of formal and informal care are collected at each assessment for the previous 3 months.

All caregiver domains (except the qualitative interviews) are measured at $T_{0}$ (after enrollment), $T_{1}$ ( 3 months after $T_{0}$ ), and $T_{2}\left(6\right.$ months after $T_{0}$ ) via telephone interview. Assessors are trained $\mathrm{PhD}$ students or research assistants who are blind to the treatment condition. Caregivers are provided with some of the questionnaires to make the interviews easier for them.

\section{Care counsellors}

As further secondary outcomes we assess counsellor's self-efficacy with questions from the Counselor Activity Self-Efficacy Scales (CASES) - subscale 'Session Management Self-Efficacy' [33] and the Counselling SelfEstimate Inventory (COSE) - subscale 'Difficult Client Behaviors' [34]. Additional measures are the Maslach

Table 1 Primary and secondary outcomes collected at the three time points

\begin{tabular}{|c|c|c|c|c|}
\hline Measures & Tools & $\mathrm{T}_{0}$ & $\mathrm{~T}_{1}$ & $\mathrm{~T}_{2}$ \\
\hline \multicolumn{5}{|l|}{ Primary outcome (caregivers) } \\
\hline Depressive symptoms & CES-D & $x$ & $x$ & $x$ \\
\hline \multicolumn{5}{|l|}{ Secondary outcomes (caregivers) } \\
\hline Caregiver burden & SCQ (short version) & $x$ & $x$ & $x$ \\
\hline Subjective physical complaints & GBB-24 (subscale: Pains in Limbs) & $x$ & $x$ & $x$ \\
\hline Negative Problem Orientation & SPSI-R (subscale: Negative Problem Orientation) & $x$ & $x$ & $x$ \\
\hline Leisure Time Satisfaction & LTS & $x$ & $x$ & $x$ \\
\hline Secondary outcomes (care counsellors) & & $\mathrm{T}_{0}^{*}$ & $\mathrm{~T}^{*}{ }_{1}$ & $\mathrm{~T}^{*}{ }_{2}$ \\
\hline Session management self-efficacy & CASES (subscale: Session Management Self-Efficacy) & $x$ & $x$ & $x$ \\
\hline Difficult client behaviors self-efficacy & COSE (subscale: Difficult Client Behaviors) & $x$ & $x$ & $x$ \\
\hline
\end{tabular}

Abbreviations: CASES counselor activity self-efficacy scales, CES-D centre for epidemiological studies depression scale, COSE counseling self-estimate inventory, GBB-24 Giessen subjective complaints list, LTS leisure time satisfaction questionnaire, SCQ sense of competence questionnaire, SPSI-R social problem solving inventory - revised

Measurement points: $T_{0}=$ baseline, $T_{1}=3$ months, $T_{2}=6$ months, $T^{*}{ }_{0}=$ basline, $T^{*}{ }_{1}=6$ months, $T^{*}{ }_{2}=12$ months 
Burnout Inventory (MBI) with the subscales 'Emotional Exhaustion', 'Depersonalization', and 'Personal Accomplishment' [35, 36], a check-list for self-care [37], and the Trier Inventory of Chronic Stress (TICS) - subscale 'Work Overload' [38]. Furthermore, the quality of counselling is rated by the informal caregivers at $T_{1}$ and $T_{2}$.

Counsellors assigned to the intervention group are evaluated at $\mathrm{T}^{*}{ }_{0}$ (before the training), at $\mathrm{T}^{*}{ }_{1}$ after finishing the training and before the evaluation of their clients (6 months after $\left.\mathrm{T}^{*}{ }_{0}\right)$, and at $\mathrm{T}_{2}{ }_{2}\left(12\right.$ months after $\left.\mathrm{T}^{*}\right)$. Counsellors assigned to control group are evaluated at $\mathrm{T}_{0}^{*}$ (before evaluation of their clients), $\mathrm{T}^{*}{ }_{1}$ (before training: 6 months after $\mathrm{T}^{*}{ }_{0}$ ), $\mathrm{T}^{*}{ }_{2}$ (after the training: 12 months after $\left.\mathrm{T}^{*}{ }_{0}\right)$. The counsellors receive the paperand-pencil questionnaires with their code number by post.

\section{Sample size calculation}

In a first step we conducted an a priori power analysis based on procedures from Borm, Fransen, and Lemmens [39] for analysis of covariance in randomized clinical trials. To detect an expected minimum effect size of .40 (Cohen's d) after the 12-month period with power $\beta$ $=.80$ (two-sided test, $\alpha=.05$ ), assuming a correlation $\rho$ $=.70$ between baseline and follow-up assessments, 52 participants in each group are needed. Because of the reduced power within a cluster-randomized design the number of participants $(n=104)$ was multiplied with a design factor (DE), that was calculated with the formula: $\mathrm{DE}=1+\mathrm{ICC}^{*}(\mathrm{~m}-1) \quad(\mathrm{DE}=$ Design $\quad$ effect,$\quad \mathrm{ICC}=$ intra cluster correlation coefficient, $\mathrm{m}=$ number of caregivers per cluster). Each counsellor represents a cluster who recruits six caregivers $(m=6)$. The estimation of the ICC $(\mathrm{ICC}=0.138$ ) was based on previous data (Pfeiffer et al., [12]). By multiplication of the design effect by the calculated sample size without cluster effect $(n=104)$, a sample size of 88 caregivers in each group was obtained. Because we assumed that not all counsellors will recruit 6 caregivers, the target number for participating counsellors was $44(20 * 6+10 * 4+8 * 2+6 * 0$, while the first factors represents the "number of counsellors", and the second factors the assumed "number of caregivers recruited by each counsellor").

\section{Statistical methods}

The comparability of the care counsellors and caregivers in the intervention and control groups are analyzed with independent samples t-tests on continuous variables, Mann-Whitney $U$ on ordinal or continuous, but not normally distributed variables, and $\chi^{2}$ tests or Fisher's exact tests of independence for categorical variables. Because of the fact that in this cluster RCT the number of randomized local offices and care counsellors is much smaller than the number of participating caregivers who are the unit of analyses we assume an increased likelihood of imbalance between caregivers in the intervention and control conditions on baseline characteristics. Such possible imbalances at baseline threaten the validity of inferences regarding intervention effects unless an appropriate statistical adjustment is used [40]. Therefore counsellors and caregivers are matched on propensity scores using the optimal matching approach before conducting treatment effectiveness analyses. Subsequent to the matching we compare the original sample with the matched sample. Treatment effects are tested with confirmatory endpoint analyses (analysis of covariance/ ANCOVA; two sided 5\% level) after three $\left(\mathrm{T}_{2}\right)$ and 6 months $\left(\mathrm{T}_{3}\right)$, using baseline scores as a covariate. For the intend-to-treat (ITT) analysis we use maximumlikelihood multiple imputation to impute missing values for withdrawn subjects or participants with missing data at month 3 and 6 . The continuous longitudinal outcome data will be analyzed by random coefficient models to apply on the patient-specific time series, and, for purposes of confirmation, by an ANOVA with repeated measures. Mean effects per care counselor are compared between intervention and control condition.

\section{Discussion}

The aim of this approach is to train caregiver counsellors to address specifically caregiver issues in addition to the recent care counselling routine. In our translation approach together with three LTC insurances with more than 9 million members we evaluate the effectiveness of a modified previously proven intervention approach [12] in this routine setting. As in previously published intervention studies [9], modifications have to be made to simplify our original intervention protocol to fit the delivery environment and resources of the LTC insurances. We could define with the participating LTC insurances only a minimum intensity for the additional counselling component that is well below the intensity in the successful efficacy trial [12]. It is unclear if this minimum dosage is sufficient to yield any effects on caregiver depression, while at the same time we do not know if the combination of our intervention component with the already existing statutory care counselling yields to synergies. Further crucial issues could be the counsellors' adherence to the defined screening schedule and the proper delivery of the intervention. Possible moderators for the adherence could be lacking time for extra work, lacking gratification, but also the circumstance that some counsellors only participate in the study due to their supervisors' directive. While all participating counsellors have a comparable specific training, the influence of their different original professions as social workers, nurses or social insurance employees on the delivery of the intervention is unclear. It is also possible that the 
counsellors of the control condition will provide a better counselling when they know they get evaluated. Furthermore, new laws and regular directives within the LTC insurances during the study might influence routine procedures as well as the workload of the participating counsellors.

\section{Abbreviations}

ANOVA: Analyses of variance; CG: Caregiver; ICC: Intra cluster correlation coefficient; ISRCTN: International Standard Randomised Controlled Trial Number; LTC: Long term care; m: Number of caregivers per cluster; $n:$ Number

of participants; PLiP: ProblemLösen in der Pflegeberatung [Problem-solving in caregiver-counselling]; PS: Problem-solving; RCT: Randomized controlled trial; ref:: Reference; SGB: Social security code; $T_{0}, T_{1}, T_{2}$ : Measurement points, $\mathrm{T}_{0}=$ baseline, $\mathrm{T}_{1}=3$ months follow-up, $\mathrm{T}_{2}=6$ months follow-up

\section{Acknowledgements}

The authors thank Anna Pendergrass who is now at the Friedrich-AlexanderUniversity Erlangen-Nuremberg for help with initial study planning, recruitment and evaluation. In addition, we thank Katrin Török (AOK Baden-Wuerttemberg), Marianna Hanke-Ebersoll (AOK Bavaria), and Dirk Ender (Social Insurance for Agriculture, Forestry and Horticulture) from the participating LTC insurances for their support.

\section{Funding}

The study is funded by the National Association of Statutory Health Insurance Funds (GKV-Spitzenverband, Germany) from May 2013 to October 2016. It is part of the programme 'Model projects for further development of the statutory German nursing care insurance according to § 8.3 SGB XI (Modellvorhaben zur Weiterentwicklung der Pflegeversicherung gem. § 8 Abs.3 SGB XI).

\section{Availability of data and material}

Not applicable for data. Further information on the card-sorting task and worksheets used in the intervention are available from the corresponding author on reasonable request.

\section{Authors' contributions}

$\mathrm{KP}$ is the principal investigator of the study and participated in developing the study and intervention protocol. MH participated in the development of the intervention protocol and was responsible for the evaluation. $\mathrm{CB}$ contributed to the study protocol and the cooperation with the participating nursing care insurances. MP and JG were involved in developing and conducting the counsellor training and supervision, DA the evaluation. KP and DA mainly drafted the manuscript. All authors revised and approved the submitted manuscript.

\section{Competing interests}

The authors declare that they have no competing interests.

\section{Consent for publication}

Not applicable.

\section{Ethics approval and consent to participate}

This research was approved in Germany by the Ethics Committee of the University of Tuebingen (17/10/2013, ref.: 508/2013BO2) and retrospectively registered at ISRCTN registry (ISRCTN23635523; www.isrctn.com). All participants (counsellors and informal caregivers) signed an informed consent form prior to participation.

\section{Author details}

'Department of Clinical Gerontology and Rehabilitation,

Robert-Bosch-Hospital, Auerbachstr. 110, 70376 Stuttgart, Germany. ${ }^{2}$ Department of Psychology, Clinical Psychology and Psychotherapy, Eberhard Karls University, Schleichstr. 4, 72076 Tuebingen, Germany.
Received: 3 November 2016 Accepted: 1 March 2017

Published online: 06 March 2017

\section{References}

1. Statistisches Bundesamt [The Federal Statistical Office] (Ed.). "Pflegestatistik 2013. Pflege im Rahmen der Pflegeversicherung. Deutschlandergebnisse. [Care statistics 2013. Care in the context of the long-term care insurance. Nationwide findings]." Wiesbaden; 2015.

2. Pinquart M, Sörensen S. Differences between caregivers and noncaregivers in psychological health and physical health: a meta-analysis. Psychol Aging. 2003;18(2):250-67.

3. Adelman RD, Tmanova LL, Delgado D, Dion S, Lachs MS. Caregiver burden: a clinical review. JAMA. 2014;311(10):1052-60.

4. Vandepitte S, Van Den Noortgate N, Putman K, Verhaeghe S, Faes K, Annemans $L$. Effectiveness of supporting informal caregivers of people with dementia: a systematic review of randomized and non-randomized controlled trials. J Alzheimers Dis JAD. 2016;52(3):929-65.

5. Bakas T, et al. Evidence for stroke family caregiver and dyad interventions: a statement for healthcare professionals from the American Heart Association and American Stroke Association. Stroke J Cereb Circ. 2014;45(9):2836-52.

6. Sörensen $S$, Pinquart $M$, Duberstein $P$. How effective are interventions with caregivers? An updated meta-analysis. The Gerontologist. 2002;42(3):356-72.

7. Pendergrass A, Becker C, Hautzinger M, and Pfeiffer K. "Dementia caregiver interventions: a systematic review of caregiver outcomes and instruments in randomized controlled trials." Int J Emerg Ment Health Hum Resil. 2015; 17(2):459-68.

8. Gitlin L, Hodgson N. Caregivers as therapeutic agents in dementia care: the context of caregiving and the evidence base for interventions. In: Gaugler $J E$, Kane RL, editors. Family caregiving in the new normal. Philadelphia: Elsevier; 2015. p. 305-53.

9. Gitlin LN, Marx K, Stanley IH, Hodgson N. Translating evidence-based dementia caregiving interventions into practice: state-of-the-science and next steps. The Gerontologist. 2015;55(2):210-26.

10. Nichols LO, Martindale-Adams J, Burns R, Zuber J, Graney MJ. REACH VA moving from translation to system implementation. The Gerontologist. 2016;56(1):135-44

11. GKV-Spitzenverband [National Association of Statutory Health Insurance Funds] (Ed.). "Pflegeberatung [Care counselling]." Hürth, Germany; 2012.

12. Pfeiffer $\mathrm{K}$, et al. Telephone-based problem-solving intervention for family caregivers of stroke survivors: a randomized controlled trial. J Consult Clin Psychol. 2014;82(4):628-43.

13. Cheng HY, Chair SY, Chau JP-C. The effectiveness of psychosocial interventions for stroke family caregivers and stroke survivors: a systematic review and meta-analysis. Patient Educ Couns. 2014;95(1):30-44.

14. Beinart N, Weinman J, Wade D, Brady R. Caregiver burden and psychoeducational interventions in Alzheimer's disease: a review. Dement Geriatr Cogn Disord Extra. 2012;2(1):638-48.

15. D'Zurilla TJ, Nezu A. Problem-solving therapy: a positive approach to clinical intervention. 3rd ed. New York: Springer; 2006.

16. D'Zurilla TJ, Goldfried MR. Problem solving and behavior modification. J Abnorm Psychol. 1971;78(1):107-26.

17. D'Zurilla TJ, Nezu AM, Maydeu-Olivares A. Social problem solving: theory and assessment. In: Chang EC, D TJ, Sanna LJ, editors. Social problem solving: theory, research, and training. Washington: American Psychological Association; 2004. p. 11-27.

18. Elliott TR, Shewchuk R. Problem-solving therapy for family caregivers of persons with severe physical disabilities. In: Radnitz C, editor. Cognitive-behavioral therapy for persons with disabilities. Northvale: Jason Aronson; 2000. p. 309-27.

19. Kurylo MF, Elliott TR, Shewchuk RM. FOCUS on the family caregiver: a problem-solving training intervention. J Couns Dev. 2001;79(3):275-81.

20. Ellis A, Hoellen B. Grundlagen und Methoden der Rational-emotiven Verhaltenstherapie [Principles and methods of rational-emotive behavioral therapy]. 2nd ed. Stuttgart: Klett-Cotta; 2008.

21. Radloff LS. The CES-D scale: a self-report depression scale for research in the general population. Appl Psychol Meas. 1977;1(3):385-401.

22. Hautzinger M, Bailer M, Hofmeister D, Keller F. Allgemeine Depressionsskala (ADS) Manual. [General Depression Scale (GDS) Manual]. Göttingen: Hogrefe; 2012.

23. Craig TJ, Van Natta PA. Current medication use and symptoms of depression in a general population. Am J Psychiatry. 1978;135(9):1036-9.

24. Pendergrass $A$, Beische $D$, Becker $C$, Hautzinger M, Pfeiffer K. An abbreviated German version of the sense of competence questionnaire among informal 
caregivers of relatives who had a stroke: development and validation. Eur J Ageing. 2015;12(3):203-13.

25. M. J. F. J. Vernooij-Dassen. Dementie en thuiszorg: Een onderzoek naar determinenten van het competentiegevoel van centrale verzorgers en het effect van professionele interventie [Dementia and home care: Determinants of the sense of competence of primary caregivers and the effect of professionally guided caregiver support]. Lisse: Swets \& Zeitlinger; 1993.

26. Brähler E, Hinz A, Scheer JW. Gießener Beschwerdebogen (GBB-24) [Giessener Symptom List (GBB-24)]. Bern: Huber; 2008.

27. D'Zurilla TJ, Nezu AM, Maydeu-Olivares A. Social Problem-Solving InventoryRevised (SPSI-R). North Tonawanda: Multi-Health Systems; 2002.

28. Graf A. Psychometrische Überprüfung einer deutschsprachigen Übersetzung des SPSI-R [Psychometric testing of a German translation of the SPSI-R]. Z Für Differ Diagn Psychol. 2003;24(4):277-91.

29. A. B. S. Director, et al. Measurement of leisure time satisfaction in family caregivers. Aging Ment Health. 2004;8(5):450-9

30. Brouwer WBF, van Exel NJA, van Gorp B, Redekop WK. The CarerQol instrument: a new instrument to measure care-related quality of life of informal caregivers for use in economic evaluations. Qual Life Res. 2006; 15(6):1005-21.

31. Mahoney Fl, Barthel DW. Functional evaluation: The Barthel Index. Md State Med J. 1965;14:61-5.

32. Prosiegel M, Böttger S, Schenk T. Der Erweiterte Barthel-Index (EBI) - eine neue Skala zur Erfassung von Fähigkeitsstörungen bei neurologischen Patienten. J Neurol Rehabil. 1996;2:7-13.

33. Lent RW, Hill CE, Hoffman MA. Development and validation of the counselor activity self-efficacy scales. J Couns Psychol. 2003;50(1):97-108.

34. Larson LM, Suzuki LA, Gillespie KN, Potenza MT, Bechtel MA, Toulouse AL. Development and validation of the counseling self-estimate inventory. J Couns Psychol. 1992;39(1):105-20.

35. Büssing A, Perrar K-M. Die Messung von Burnout. Untersuchung einer deutschen Fassung des Maslach Burnout Inventory (MBI-D). [Measuring burnout: A study of a German version of the Maslach Burnout Inventory (MBI-D).]. Diagnostica. 1992;38(4):328-53.

36. Kaschka WP, Korczak D, Broich K. Burnout: a fashionable diagnosis. Dtsch Arzteblatt Int. 2011;108(46):781-7.

37. Kaluza G. Stressbewältigung. Trainingsmanual zur psychologischen Gesundheitsförderung [Stress management. A manual for psychological health promotion]. Berlin: Springer; 2011.

38. Schulz P, Schlotz W, Becker P. TICS. Trierer Inventar zum chronischen Stress [TICS. Trier Inventory for Chronic Stress]. Göttingen: Hogrefe; 2004.

39. Borm GF, Fransen J, Lemmens WAJG. A simple sample size formula for analysis of covariance in randomized clinical trials. J Clin Epidemiol. 2007; 60(12):1234-8.

40. Leon AC, Demirtas H, Li C, Hedeker D. Subject-level matching for imbalance in cluster randomized trials with a small number of clusters. Pharm Stat. 2013;12(5):268-74.

\section{Submit your next manuscript to BioMed Central and we will help you at every step:}

- We accept pre-submission inquiries

- Our selector tool helps you to find the most relevant journal

- We provide round the clock customer support

- Convenient online submission

- Thorough peer review

- Inclusion in PubMed and all major indexing services

- Maximum visibility for your research

Submit your manuscript at www biomedcentral.com/submit

) Biomed Central 